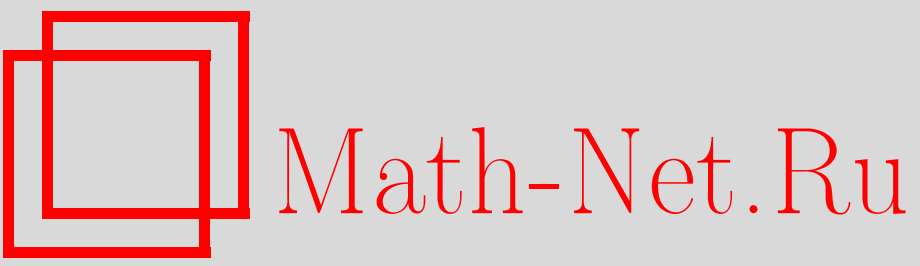

А. В. Селиванов, О заменах времени для процессов Леви, УМН, 2003, том 58, выпуск 2, 175-176

DOI: https://doi.org/10.4213/rm623

Использование Общероссийского математического портала Math-Net.Ru подразумевает, что вы прочитали и согласны с пользовательским соглашением

http://www . mathnet.ru/rus/agreement

Параметры загрузки:

IP : 18.207 .199 .55

26 апреля 2023 г., 11:24:03 


\section{О ЗАМЕНАХ ВРЕМЕНИ ДЛЯ ПРОЦЕССОВ ЛЕВИ}

\section{А. В. СЕливанов}

В данной заметке рассматривается следующая задача. Пусть на вероятностном пространстве $(\Omega, \mathscr{F}, Q)$ заданы процесс Леви $Z$ и неотрицательньй неубывающий càdlàg процесс $\tau$, независимый с $Z$. Положим $X:=Z \circ \tau$, т.е. $X_{t}=Z_{\tau_{t}}, t \geqslant 0$. Поставим вопрос о локальной абсолютной непрерывности меры $\widetilde{P}:=\operatorname{Law}\left(X_{t}, t \geqslant 0\right)$ относительно меры $P:=\operatorname{Law}\left(Z_{t}, t \geqslant 0\right)$. Эти меры считаем заданными на пространстве Скорохода $D\left(\mathbb{R}_{+}\right)$с канонической фильтрацией $\left(\mathscr{F}_{t}\right)_{t \geqslant 0}$.

Если $Z$ не является составным пуассоновским процессом, то, как вытекает из $[1 ; 30.1,33.1]$ и [2; теорема 2$]$, в нетривиальной ситуации нельзя ожидать свойства локальной абсолютной непрерывности меры $\widetilde{P}$ относительно $P$. Далее, легко показать, что если $\tau$ - детерминированный не абсолютно непрерьвньй процесс, то $\widetilde{P} \stackrel{\text { loc }}{K} P$.

ТеОРема 1. Пусть $Z$ - составной пуассоновский процесс, $\tau$ абсолютно непрерывен. Тогда $\widetilde{P} \stackrel{\text { loc }}{\ll} P$.

Для доказательства этой теоремы нам потребуется следующее утверждение, представляющее самостоятельньй интерес.

Теорема 2. Пусть $Z$ - составной пуассоновский процесс с мерой Леви $\nu, \tau$ абсолютно непрерывен, $\tau_{t}=\int_{0}^{t} a_{s} d s$. Тогда компенсатор прочесса $Z \circ \tau$ относительно фильтрации $\mathscr{F}_{t}^{Z \circ \tau}:=\bigcap_{\varepsilon>0} \sigma\left(Z_{\tau_{s}}, 0 \leqslant s \leqslant t+\varepsilon\right)$ имеет вид $\mathrm{E}\left(a_{t} \mid \mathscr{F}_{t}^{Z \circ \tau}\right)(\omega) d t \nu(d x) c$ измеримой по паре $(t, \omega)$ версией условного математического ожидания.

ДокАЗАтЕльство. Существование измеримой по паре $(t, \omega)$ версии $\mathrm{E}\left(a_{t} \mid \mathscr{F}_{t}^{Z \circ \tau}\right)(\omega)$ доказано в [3], [4]. Обозначим меру $\mathrm{E}\left(a_{t} \mid \mathscr{F}_{t}^{Z \circ \tau}\right)(\omega) d t \nu(d x)$ через $\eta(\omega ; d t, d x)$. Покажем, что $\eta-$ предсказуемая мера. Действительно, для любого $t \geqslant 0$ и любой неотрицателшной предсказуемой функции $W(\omega, t, x)$

$$
(W * \eta)_{t}(\omega)=\int_{(0, t] \times \mathbb{R}} W(\omega, s, x) \eta(\omega ; d s, d x)=\int_{(0, t]}\left[\int_{\mathbb{R}} W(\omega, s, x) \nu(d x) \mathrm{E}\left(a_{s} \mid \mathscr{F}_{s}^{Z \circ \tau}\right)(\omega)\right] d s,
$$

если интегралы конечны. В этом случае выражение в квадратных скобках $\left(\mathscr{B}\left(\mathbb{R}_{+}\right) \times \mathscr{F}{ }_{S}^{Z \circ \tau}\right)$-измеримо, а $(W * \eta)_{t}$ имеет непрерьвные траектории, что влечет предсказуемость меры $\eta$.

Далее, обозначим через $\mu$ меру скачков процесса $Z \circ \tau$ :

$$
\mu(\omega ; d t, d x):=\sum_{s \geqslant 0} I\left(\Delta Z_{\tau_{s}} \neq 0\right) \delta_{\left(s, \Delta Z_{\tau_{s}}\right)}(d t, d x) .
$$

Проверим, что

$$
\mathrm{E}(W * \mu)_{\infty}=\mathrm{E}(W * \eta)_{\infty}
$$

для всякой неотрицательной предсказуемой функции $W=W(\omega ; t, x)$. В силу теоремы о монотонных классах достаточно рассмотреть функции $W$ вида $W(\omega ; t, x)=I_{(u, v] \times B}(t, x) f\left((Z \circ \tau) u_{1}\right.$, $\left.\ldots,(Z \circ \tau) u_{n}\right)$, где $n \in \mathbb{N}, B \in \mathscr{B}(\mathbb{R}), 0 \leqslant u_{1} \leqslant \cdots \leqslant u_{n} \leqslant u<v, f: \mathbb{R}^{n} \rightarrow \mathbb{R}_{+}$- борелевская функция. Тогда

$$
\mathrm{E}(W * \mu)_{\infty}=\mathrm{E} f\left((Z \circ \tau) u_{1}, \ldots,(Z \circ \tau) u_{n}\right) \sum_{u<s \leqslant v} I\left(\Delta Z_{\tau_{s}} \neq 0\right) I\left(\Delta Z_{\tau_{s}} \in B\right) .
$$

В силу независимости процессов $Z$ и $\tau$ можно считать, что $(\Omega, \mathscr{F}, \mathrm{Q})=\left(\Omega_{1}, \mathscr{F}_{1}, \mathrm{Q}_{1}\right) \times$ $\left(\Omega_{2}, \mathscr{F}_{2}, \mathrm{Q}_{2}\right)$, где процесс $Z$ задан на $\Omega_{1}$, а $\tau$-на $\Omega_{2}$. Далее, воспользовавшись явным построением составного пуассоновского процесса, можно считать, что $Z_{t}=\sum_{k=1}^{\pi_{t}} \xi_{k}$, где $\pi$-пуассоновский 
процесс с параметром $\nu(\mathbb{R}),\left\{\xi_{k}\right\}_{k=1}^{\infty}-$ независимые одинаково распределенные случайные величины с распределением $\nu / \nu(\mathbb{R})$, независимые с $\pi$. Тогда

$$
\begin{aligned}
& \mathrm{E}(W * \mu)_{\infty}=\mathrm{E}_{2}\left[\mathrm{E}_{1} f\left(Z_{\tau_{u_{1}}\left(\omega_{2}\right)}\left(\omega_{1}\right), \ldots, Z_{\tau_{u_{n}}\left(\omega_{2}\right)}\left(\omega_{1}\right)\right) \sum_{u<s \leqslant v} I\left(\Delta Z_{\tau_{s}\left(\omega_{2}\right)}\left(\omega_{1}\right) \in B \backslash\{0\}\right)\right] \\
& \stackrel{\mathrm{a})}{=} \mathrm{E}_{2}\left[\mathrm{E}_{1} f(\ldots) \cdot \mathrm{E}_{1} \sum_{u<s \leqslant v} I(\ldots)\right]=\mathrm{E}_{2}\left[\mathrm{E}_{1} f(\ldots) \cdot \mathrm{E}_{1} \sum_{k=\pi_{\tau_{u}\left(\omega_{2}\right)}}^{\pi_{\tau_{v}\left(\omega_{2}\right)}} I\left(\xi_{k} \in B\right)\right] \\
& =\mathrm{E}_{2}\left[\mathrm{E}_{1} f(\ldots) \cdot P\left(\xi_{k} \in B\right) \mathrm{E}_{1}\left(\pi_{\tau_{v}\left(\omega_{2}\right)}-\pi_{\tau_{u}\left(\omega_{2}\right)}\right)\right] \\
& =\mathrm{E}_{2}\left[\mathrm{E}_{1} f(\ldots) \cdot \frac{\nu(B)}{\nu(\mathbb{R})} \nu(\mathbb{R})\left(\tau_{v}-\tau_{u}\right)\right]=\mathrm{E}\left[f(\ldots) \nu(B) \int_{u}^{v} a_{s} d s\right] \\
& =\mathrm{E}\left[\mathrm{E}\left(f(\ldots) \nu(B) \int_{u}^{v} a_{s} d s \mid \mathscr{F}_{u}^{Z \circ \tau}\right)\right] \stackrel{\mathrm{b})}{=} \mathrm{E}\left[f(\ldots) \nu(B) \int_{u}^{v} \mathrm{E}\left(a_{s} \mid \mathscr{F}_{u}^{Z \circ \tau}\right) d s\right] \\
& =\mathrm{E}\left[\mathrm{E}\left(f(\ldots) \nu(B) \int_{u}^{v} \mathrm{E}\left(a_{s} \mid \mathscr{F}_{s}^{Z \circ \tau}\right) d s \mid \mathscr{F}_{u}^{Z \circ \tau}\right)\right]=\mathrm{E}(W * \eta)_{\infty}
\end{aligned}
$$

где $\mathrm{E}_{i}, i=1,2$, - математические ожидания на пространстве $\left(\Omega_{i}, \mathscr{F}_{i}, \mathrm{Q}_{i}\right)$; в а) мы воспользовались независимостью приращений процесса $Z_{\tau_{t}\left(\omega_{2}\right)}$ для любого фиксированного $\omega_{2} ;$ в b) применена теорема Фубини и тот факт, что $f\left(Z_{\tau_{u_{1}}}, \ldots, Z_{\tau_{u_{n}}}\right)$ есть $\mathscr{F}_{u}^{Z \circ \tau}$-измеримая величина. Заметим, что в силу неотрицательности выражений под знаком математических ожиданий $\mathrm{E}(W * \eta)_{\infty}$ и $\mathrm{E}(W * \mu)_{\infty}$ вместе либо конечны, либо бесконечны.

Итак, равенство (1) проверено, и тем самым установлено утверждение теоремы 2.

Теперь теорема 1 является следствием из общей теории интегралов Хеллингера, а именно, из [5; IV.4.16] с учетом [5; I.3.13]. Заметим, что из [5; IV.4.16] с помощью теоремы 2 легко следует резултат о взаимном положении самих мер $\widetilde{P}$ и $P$.

ЗАмечАниЕ. Теорема 2 показывает, что даже если случайная величина $a_{t}$ не имеет первого момента, то $\mathrm{E}\left(a_{t} \mid \mathscr{F}_{t}^{Z \circ \tau}\right)$ конечно для $(\mathrm{Q}(d \omega) \times d t)$-п.в. $(\omega, t)$.

ЗАмЕчАниЕ. Легко доказать, что компенсатор процесса $Z \circ \tau$ относителшно произволњной фильтрации $(\mathscr{F} t)_{t \geqslant 0}$, с которой согласованы процессы $Z \circ \tau$ и $\tau$, есть $a_{t}(\omega) d t \nu(d x)$. Таким образом, теорема 2 является в некотором смысле аналогом теоремы об обновляющих процессах (см. [6; теорема 7.12]).

В заключение автор хотел бы выразить глубокую благодарность и признательность к.ф..-м.н. А. С. Черному за огромную помощь и научное руководство.

\section{СПИСОК ЛИТЕРАТУРЫ}

[1] K.-I. Sato. Lévy Processes and Infinitely Divisible Distributions. Cambridge: Cambridge Univ. Press, 1999. [2] P. L. Brockett, H. G. Tucker // J. Multivariate Anal. 1977. V. 7. № 1. Р. 13-27. [3] М.П. Ершов // Теория вероятн. и ее примен. 1972. Т. 17. №1. С. 167-172. [4] П. А. Мейер. Вероятность и потенциалы. М.: Мир, 1973. [5] ЖК. Жакод, А. Н. Ширяев. Пределшные теоремы для случайных процессов. Т. 1. М.: Физматлит, 1994. [6] Р .Ш. Липцер, А. Н. Ширяев. Статистика случайных процессов. М.: Наука, 1974. 